(c) EIsevier/INRA/DIB/AGIB

\title{
Morphologischer Vergleich von Völkern der "Land- biene" in Niedersachsen mit typischer Apis mellifera carnica und Apis mellifera mellifera
}

\author{
N Reinsch 1, H Schuster 1, K Bienefeld 2, F Pirchner 1 \\ 1 Institut für Tierzucht, TU München-Weihenstephan D-8050 Freising; \\ 2 Institut für Bienenkunde, (Polytechnische Gesellschaft), Fachbereich Biologie der \\ JW Goethe-Universität, Karl-von-Frisch-Weg 2, D-6370 Oberursel, Deutschland
}

(Eingegangen 6 Oktober 1990; angenommen 24 November 1990)

Zusammenfassung - Aus 99 Völkern in Niedersachsen wurden Bienenproben entnommen und morphologish untersucht. Keines der Völker entsprach voll dem Rassestandard für Carnica des Deutschen Imkerbundes. Mittels Hauptkomponenten- und Diskriminanzanalyse konnte gezeigt werden, daß die niedersächsische "Landbienen"-Population der Carnica-Rasse sehr viel ähnlicher ist als der Mellifera-Rasse, obwohl noch ein deutlicher Mellifera-Einfluß festzustellen ist.

Apis mellifera carnica / Apis mellifera mellifera / Morphometrie / Verdrängungszucht

\section{EINLEITUNG}

Seit mehr als 40 Jahren werden in Deutschland Carnica-Bienen gezüchtet, mit dem Ziel, die weniger leistungstähige einheimische Mellifera zu verdrängen. Unser Ziel war es, anhand morphologischer Merkmale zu klären, ob und wie sehr sich die Völker der heutigen Niedersächsischen "Landrasse" von typischen Völkern der beiden genannten Rassen unterscheiden.

\section{MATERIAL UND METHODEN}

Die benötigten Bienen wurden im April 1988 auf 33 Bienenständen im nordöstlichen Niedersachsen gesammelt (Gebiet zwischen Hamburg-Hannover-Bremen-Cuxhaven). Drei der 33 Imker waren Reinzüchter, alle anderen hat- ten sich noch nie, oder schon seit Jahren nicht mehr, mit Zuchtarbeit befaßt. Bei jedem der Imker wurden von drei Völkern Bienenproben gezogen. Die Königinnen dieser Völker waren bis auf eine Ausnahme standbegattet. In 47 Fällen stammte die Mutter der Königin aus einem nicht näher bezeichneten Volk vom eigenen Stand, in 29 Fällen wurde die Königin des untersuchten Volkes vom Imker als Tochter einer Carnica-Königin eingestuft und fünfmal waren Nachkommen solcher Königinnen vertreten. Bei den restlichen 18 Völkern waren Herkunft und Begattung unsicher ( $z T$ auch Schwärme). Gekörte Carnica-Zuchtvölker waren also nicht vertreten, sondern nur Völker, die nach Abstammung und Begattung ihrer Königin als Vertreter der "Landrasse" einzustufen sind. Dieser Teil der niedersächsischen Bienenpopulation wird von der organisierten Zuchtarbeit nicht direkt erfaßt. Je Volk wurden 50 Arbeiterinnen entnommen. Da bei 6 Völkern einige Arbeiterinnen unbrauchbar waren, konnten insgesamt 4853 Bienen untersucht werden. An jeder Biene wurden die folgenden Merkmale 
gemessen: Cubitalindex, Rückenhaarlänge, und Filzbindenbreite.

Der Cubitalindex wurde mit Mikrofilmlesegerät und Indexfächern gemesen (Schuster et al, 1989). Für jede Biene wurde neben dem Meßwert festgehalten, ob ihr Cubitalindex im Bereich unter 2,0 oder zwischen 1,86 und 2,33 lag. Die Rückenhaarlänge wurde in die drei Klassen kurz, mittel und lang eingeteilt, die Filzbindenbreite in die Klassen schmal, mittel und breit (Code jeweils 1,2 und 3). Diese beiden Merkmale wurden so, wie von Ruttner (1988a) beschrieben, bestimmt. Danach wurde für jedes Volk und jedes Merkmal der Mittelwert gebildet, so daß fünf verschiedene Werte je Volk berücksichtigt werden konnten: 1) mittlerer Cubitalindex (MCl), 2) Anteil Bienen mit Cubitalindex unter 2,0 (VB1), 3) Anteil Bienen mit Cubitalindex zwischen 1,86 und 2,33 (VB2), 4) mittlere Rückenhaarlänge (RHL), 5) mittlere Filzbindenbreite (FBB).

Hohe Anteile VB1 und VB2 zeigen eine starke Linksschiefe bzw Nebengipfel der IndexVariationskurve an, beides Anzeichen für Mellifera-Einkreuzung. Die obige Merkmalkombination hat sich als besonders geeignet für die Unterscheidung von unverkreuzter Carnica und Völkern mit Mellifera-Einfluß erwiesen (Ruttner, 1969). Aus der morphometrischen Bienendatenbank in Oberursel (Ruttner, 1988a) standen uns die Daten von 10 typischen Carnica- und 9 typischen Mellifera-Völkern zur Verfügung. Die geographische Herkunft der einzelnen Proben war folgende (Anzahlen in Klammern): $A$ m carnica: Österreich (5), Jugoslowien (5); A m mellifera: Isle of Man (1), Irland (2), Dänemark (1), England (2), Südnorwegen (3). Zu jeder Biene waren Cubitalindex, Rückenhaarlänge und Filzbindenindex gegeben. Die kontinuierlichen Werte für Rückenhaarlänge und Filzbindenindex wurden in Klassen umgewandelt (Ruttner, 1988a; Goetze, 1949) und für jedes Volk die gleichen Merkmale wie oben angegeben gebildet. Zur Auswertung wurden die Völker aus Niedersachsen mit den Rassenstandards des Deutschen Imkerbundes (DIB, 1988) für die Rassen Carnica und Mellifera verglichen. Diesem Standard liegen die Untersuchungen von Ruttner (1969) zugrunde. Um die Zuchbenutzung von verkreuzten Völkern mit hoher $\mathrm{Si}$ cherheit ausschließen zu können, sind die Anforderungen an reinrassige Völker im DIBStandard strenger formuliert, als es der durchschnittlichen natürlichen Carnica entspricht. Im zweiten Schritt wurden die Völker aus der Datenbank mit einbezogen und eine Hauptkomponentenanalyse sowie eine Diskriminanzanalyse (Linder und Berchtold, 1982) durchgeführt. Für alle. Berechnungen wurde das Statistikprogrammpaket SAS benutzt.

\section{ERGEBNISSE}

Die durchschnittlichen Völkermittelwerte der drei Gruppen zeigt Tabelle I. Die Niedersächsische Population unterscheidet sich in allen Merkmalen deutlich von den Mellifera-Völkern. Unterschiede zur Carnica sind in den Merkmalen Rückenhaarlänge und Filzbindenbreite zu erkennen, in den Flügelmerkmalem sind sie nur gering. Auffallend ist bei den Carnica-Völkern der etwas hohe Anteil von Beinen mit einem Cubitalindex unter 2,0.

Kein einziges der 99 Völker aus Niedersachsen konnte bei Zugrundelegung des DIB-Rassestandards als reines Carnicaoder Mellifera-Volk klassifiziert werden. Die Ausschlußgründe für Carnica waren bei 95 Völkern zu langes Rückenhaar. Der mittlere Cubitalindex war bei 53 Völkern zu niedrig, 26 mal war zwar der mittlere Cubitalindex ausreichend hoch, wurde aber die Index-Variationskurve dem Standard für Carnica nicht gerecht (zu viele Bienen in den Bereichen unter 2,0 oder zwischen 1,86 und 2,33). Zum Ausschluß führten immer mindestens zwei Gründe.

Tabelle I. Durchschnittliche Völkermittelwerte für die drei Herkünfte.

\begin{tabular}{lccc} 
Merkmal & \multicolumn{3}{c}{$\begin{array}{c}\text { Herkunft } \\
\end{array}$} \\
& Niedersachsen & Mellifera & Carnica \\
\hline MCl & 2,58 & 1,65 & 2,52 \\
VB1 & 0,08 & 0,95 & 0,06 \\
VB2 & 0,30 & 0,15 & 0,30 \\
RHL & 1,91 & 2,72 & 1,09 \\
FBB & 2,39 & 2,41 & 2,95 \\
\hline
\end{tabular}


Die gemeinsame Hauptkomponentenanalyse aller 110 Völker ergab zwei Faktoren mit einem Eigenwert von mehr als eins. Faktor 1 erfaßt $45 \%$ der Gesamtvarianz, Faktor 2 hingegen $27 \%$. Zur besseren Interpretierbarkeit wurde eine VarimaxRotation (Weber, 1974) durchgeführt. Die errechneten Faktorladungen sind in Tabelle II enthalten. Die graphische Darstellung in Abb 1 zeigt zwei deutlich getrennte Punktwolken. Die kleine Wolke rechts enthält nur Mellifera-Völker, gekennzeichnet durch " $M$ ", entsprechend deren niedrigen Cubitalindex-Werten, den langen Rückenhaaren und dem hohen Anteil von Bienen mit einem Cubitalindex unterhalb 2,0. Die große Wolke enthält rechts die Völker der niedersächsischen Population (X) und die Carnica-Völker aus der Datenbank (C) am linken Rand der Wolke. Die Carnica-Völker stellen im gesamten Material also das andere Extrem zu den Mellife- den Mellifera-Völkern ist etwa 4,5 mal so groß wie der zu den Carnica-Völkern.

\section{DISKUSSION}

Obwohl in unserer Stichprobe nur "Landbienen" (also keine Carnica-Zuchtvölker) vertreten waren, zeigt sich, daß die niedersächsische Population der Carnica sehr viel näher steht als der Mellifera, daß also eine sehr starke Verdrängung von Genen der alten dunklen Biene stattgefunden hat. Am deutlichen Kreuzungscharakter der untersuchten Völker ist zu erkennen, daß der Einfluß der zahlenmäßig kleinen Gruppe der Carnica-Reinzüchter begrenzt ist und eine weitere Annäherung der gesamten Population an den Carnica-Typ nur langsam erfolgen wird. Außer den Rassen Carnica und Mellifera bringen

Tabelle II. Faktorladungen (nach Varimax-Rotation).

\begin{tabular}{lrrrrr}
\hline & \multicolumn{3}{c}{ Merkmal } & & \\
\cline { 2 - 5 } & \multicolumn{1}{c}{ Faktor } & VBI & VB2 & RHL & FBB \\
\hline Faktor 1 & $-0,819$ & 0,952 & $-0,037$ & 0,770 & $-0,280$ \\
Faktor 2 & $-0,350$ & $-0,019$ & 0,913 & 0,120 & $-0,615$ \\
\hline
\end{tabular}

ra-Völkern dar, mit hohem Cubitalindex, kurzen Haaren und wenigen Bienen mit einem Cubitalindex unter 2,0.

Eine lineare Diskriminanzanalyse führte zu der Zuordnungsmatrix in Tabelle III. Zehn Völker der niedersächsischen Stichprobe wurden der Carnica zugeordnet, es ergibt sich also ein als Kreuzungen identifizierter Anteil von rund 90\%. Der verallgemeinerte Mahalanobis-Abstand (Linder und Berchtold, 1982) zwischen den mehrdimensionalen Gruppenmittelwerten ist in Tabelle IV angegeben. Der Abstand (linear) der niedersächsischen Population zu
Tabelle III. Anzahl der Völker, die mittels Diskriminanzanalyse den verschiedenen Gruppen zugeordnet werden.

\begin{tabular}{lrcrr}
\hline Gruppe & \multicolumn{3}{c}{ Klassifiziert als } \\
& Carnica Mellifera & $\begin{array}{c}\text { Nieder. } \\
\text { sachsen }\end{array}$ \\
& \multicolumn{5}{c}{} \\
\hline Carnica & 10 & 0 & 0 & 10 \\
Mellifera & 0 & 9 & 0 & 9 \\
Niedersachsen & 10 & 0 & 89 & 99 \\
\hline
\end{tabular}




\section{Abbildung 1}

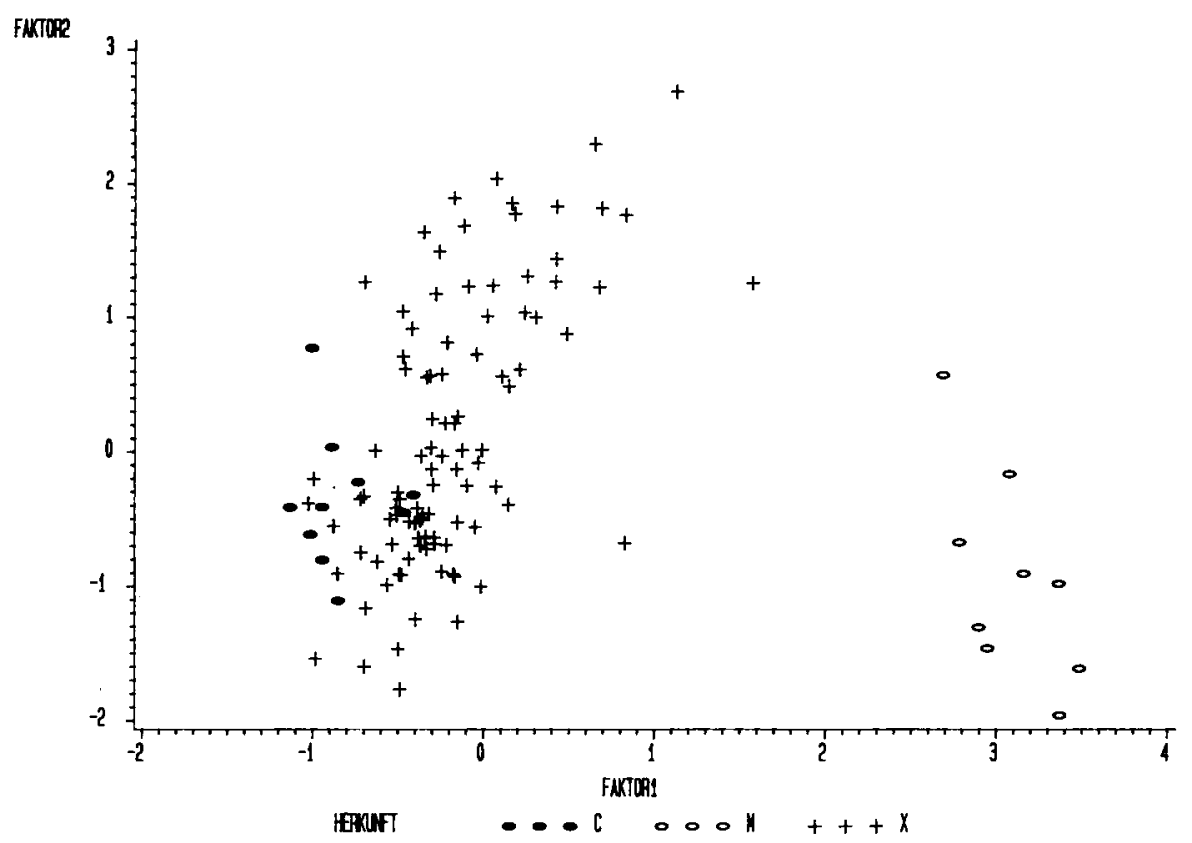

Abb 1. Graphische Darstellung einer Haupkomponentenanalyse (Faktor 1 und 2) mit 118 Proben. $C$ : A m carnica, M: A m mellifera, X: "Landrasse".

Tabelle IV. Mahalanobis-Abstände zwischen je zwei Gruppen.

\begin{tabular}{lrr}
\hline \multirow{2}{*}{ Gruppen } & \multicolumn{2}{c}{ Abstand } \\
\cline { 2 - 3 } & Quadratisch & Linear \\
\hline Niedersachsen-Carnica & 7,53 & 2,74 \\
Niedersachsen-Mellifera & 162,17 & 12,73 \\
Mellifera-Carnica & 173,62 & 13,18 \\
\hline
\end{tabular}

auch andere Herkünfte (Buckfast, Ligustica, Kaucasica) Gene in die "Landrasse" ein, so daß auch auf längere Sicht keine völlige Übereinstimmung zwischen "Landrasse" und typischer Carnica zu erwarten ist, besonders wenn andere Merkmale (z B Farbmerkmale) mit berücksichtigt werden. Ähnlich wie in Niedersachsen dürtte die Lage in Bayern sein. Bei den an die bayerischen Prüfhöfe 1985 und 1987 angelieferten Völkern lag der Anteil der Völker, der den Anforderungen an den DIB-Standard für Carnica nicht entsprach, auf den verschiedenen Prüfhöfen zwischen $3 \%$ und $46 \%$ (Mautz et al, 1990), obwohl die anliefernden Imker überdurchschnittlich stark züchterisch interessiert sind.

Eine völlige Verdrängung der MelliferaAnteile wird wohl auch dadurch erschwert, daß Wirtschaftsköniginnen oft von Mellifera-beeinflussten Drohnen standbegattet werden. Sind die Leistungen solcher Völker aufgrund geringer Inzucht und durch Heterosis gut, werden nicht wenige Imker diese Völker zur Nachzucht verwen- 
den und über längere Zeit halten, so daß die Mellifera-Gene über Königinnen und Drohnen wieder in die Population einfließen können.

Zusammenfassend kann gesagt werden, daß die Landbiene zwar noch deutliche Mellifera-Einflüsse aufweist, aber sich schon stark der Carnica angenähert hat, obwohl dieser Entwicklung einige Schwierigkeiten entgegenstehen. Wie die bayerischen Ergebnisse zeigen, dürtte in anderen Regionen des ehemaligen MelliferaGebietes die Lage ähnlich sein.

\section{DANKSAGUNGEN}

Wir möchten Herrn Dipl Ing FK Tiesler für die Unterstützung bei der Probennahme danken, sowie Herrn Prof F Ruttner und Frau Dipl Biol M Meixner für die Bereitstellung der Zahlen aus der Datenbank.

\section{Summary - Morphological comparison} of colonies of the local bee in Lower Saxony with typical $A \boldsymbol{m}$ carnica and $A$ $m$ mellifera. The current "Landrasse" (the common honeybee) of Apis mellifera in Lower Saxony (Germany) is a product of $\approx$ $40 \mathrm{yr}$ of upgrading $A m$ mellifera with $A m$ carnica. The object of this study was to make a comparison by morphometric analysis of "Landrasse" colonies with typical colonies of $A m$ mellifera and A $m$ carnica. A sample of 4853 bees from 99 colonies was taken in Lower Saxony during April 1988. Thirty-three apiaries contributed 3 colonies per apiary. Five measurements were taken from each colony:

- average of cubital index (MCl);

- proportion of bees with cubital index < 2.0 (VB1)

- proportion of bees with cubital index between 1.86-2.33 (VB2);

- average length of hair on tergite $4(R H L)$;

- average width of tomentum on the side of tergite 4 .
The last 2 measurements were coded as 1,2 and 3 (for single bees), corresponding to the classes low, medium and high (Ruttner, 1988a). The means for the colonies were computed from these codes. Data from 10 typical colonies of $A m$ carni$c a$ and 9 typical colonies of $A m$ mellifera were obtained from the morphometric bee data bank in Oberursel. The averages for each group and trait are given in table I. A comparison of the colonies from Lower Saxony with the standard for A $m$ carnica, which is based on the work of Ruttner (1969) and used by the German Beekeeper's Association (Deutscher Imkerbund), resulted in a classification of all 99 colonies as "not carnica". The graph in figure 1 was obtained by principal component analysis of all 118 colonies. The well-separated cluster on the right contains only colonies of $A m$ mellifera (M); the larger cluster on the left contains both $A m$ carnica $(C)$ and "Landrasse" $(X)$. The "Landrasse" is positioned between the extremes due to its crossbreeding character. Table II contains the coefficients for the 2 factors with eigenvalues > 1 (Varimax-rotated). After discriminant analysis, 10 "Landrasse" colonies were classified as $A m$ carnica (table III). Mahalanobis-distances between groups are shown in table IV (quadratic and linear). In conclusion, it can be stated that the "Landrasse" is much more influenced by $A$ $m$ carnica than by $A m$ mellifera in spite of clear crossbreeding characteristics such as long hair, showing the gene contribution of the native population.

\section{Apis mellifera carnica / Apis mellifera mellifera / morphometrics / upgrading}

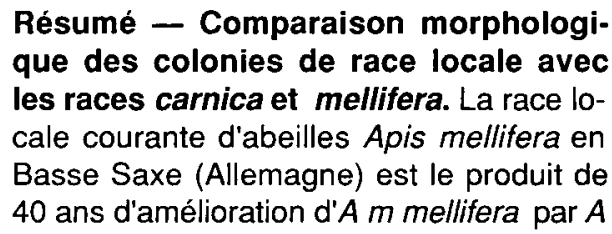


$m$ carnica. Le but de cette étude était de comparer par l'analyse morphométrique des colonies de race locale avec des colonies typiques de mellifera et de carnica. Quatre mille huit cent cinquante trois abeilles ont été prélevées en Basse Saxe courant avril 1988 dans 33 ruchers, à raison de 50 abeilles par colonie et de 3 colonies par rucher. Pour chaque colonie, 3 mesures ont été effectuées : indice cubital moyen (MCI); pourcentage d'abeilles ayant un indice cubital inférieur à 2,1 $\left(V B_{1}\right)$; pourcentage d'abeilles ayant un indice cubital compris entre 1,86 et 2,33 $\left(\mathrm{VB}_{2}\right)$; longueur moyenne des poils du $4 \mathrm{e}$ tergite (RHL); largueur moyenne du tomentum sur le côté du $4^{\mathrm{e}}$ tergite. Les 2 dernières mesures ont été codées 1,2 et 3 (pour les abeilles individuelles) suivant les classes faible, moyenne et élevée (Ruttner, 1988a). Pour les colonies, les moyennes ont été calculées d'après ces codes. La banque de données sur la morphométrie de labeille à Oberursel a fourni les données de 10 colonies typiques d' $A m$ mellifera et de 9 colonies typiques d' $A \mathrm{~m}$ carnica.

Le tableau I donne les moyennes pour chaque caractéristique et chaque groupe. De la comparaison des colonies de Basse Saxe avec la norme $A m$ carnica, basée sur le travail de Ruttner (1969) et utilisée par l'Association des apiculteurs allemands (Deutscher imkerbund), il ressort qu'aucune des 99 colonies n'est carnica. Le graphe de la figure 1 a été obtenu par analyse en composante principale des 118 colonies. Le nuage bien individualisé sur le côté droit ne contient que des colonies d'A $m$ mellifera ("M"), le nuage plus gros sur le côté gauche à la fois des $A$ m carnica ("C") et des abeilles locales ("X"). La race locale est située entre les extrêmes en raison de son caractère hybride. Le tableau II présente les coefficients pour les 2 facteurs de valeurs propres supérieures à 1 (rotation Varimax). Après analyse discriminante, 10 colonies de la race locale ont été classées en A m carnica (tableau III).

Le tableau IV donne les distances de Mahalanobis entre les groupes (distances quadratique et linéaire). En résumé, on peut dire que la race locale est beaucoup plus proche d'A $m$ carnica que d'A $m$ mellifera, malgré des caractéristiques nettes d'hybridation, telles la longueur des poils, révélant la contribution des gènes de la population autochtone.

Apis mellifera carnica / Apis mellifera mellifera / morphométrie / amélioration génétique

\section{LITERATUR}

Deutscher Imkerbund (1988) Richtlinien für das Zuchtwesen des Deutschen Imkerbundes

Goetze G (1949) Imkerliche Züchtungspraxis. Landbuch-Verlag GMBH, Hannover

Linder A, Berchtold W (1982) Statistische Methoden III. Birkhäuser, Basel

Mautz D, Kauhausen D, Moritz R (1990) Bericht über die Leistungsprüfungen an den Bayerischen Prüfhöfen seit 1985. Imkerfreund 45, 9-17

Ruttner F (1969) Biometrische Charakterisierung der österreichischen Carnica-Biene. $Z$ Bienenforsch 9, 469-503

Ruttner F (1988a) Zuchttechnik und Zuchtauslese bei der Biene. Ehrenwirth, München

Ruttner F (1988b) Biogeography and Taxonomy of Honeybees. Springer, Berlin

Schuster H, Reinsch N, Bienefeld K (1989) Vergleich verschiedener Methoden der Cubitalindex-Messung bei der Honigbiene. Allg Dtsch Imkerztg 23, 323-323

Weber $E$ (1974) Einführung in die Faktorenanalyse. VEB Gustav Fischer Verlag, Jena 